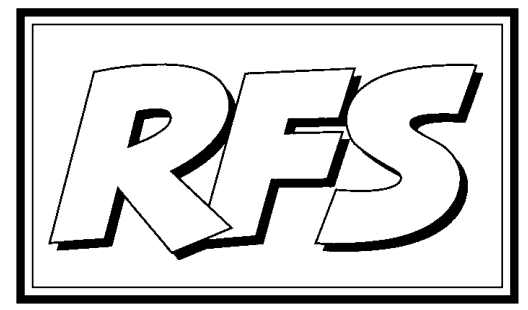

Revista de Fomento Social, 55 (2000), 161-187

\title{
El reto de lo social en la nueva Europa del tercer milenio
}

Eduardo ROJ O TORRECILLA²

\section{Consideraciones previas sobre el proceso de globalización económica y social}

Seguimos hablando diariamente de la necesidad de que el mundo del trabajo responda de forma adecuada a los cambios que se producen en el ámbito internacional de las relaciones económicas, pero todavía los deseos van muy por delante de la realidad. Y esa realidad económica que cada vez condiciona más gran parte de la actuación cotidiana de organizaciones internacionales, estados y agentes sociales, se caracteriza por un creciente incremento del proceso de internacionalización económica, que puede constatarse ya con el dato relativo a la importancia de las empresas transna-

1 El presente artículo es una versión revisada de la conferencia pronunciada el 15 de marzo de 2000 en el marco de las J ornadas organizadas por Cáritas diocesana de Salamanca sobre el jubileo 2000. Fecha de cierre del artículo: 10 de junio.

2 Catedrático de Derecho del Trabajo y de la Seguridad Social de la Universidad de Girona. Miembro del Consejo Directivo de Cristianisme i Justícia (Cij en el texto). 
cionales en este proceso: según la Comisión Europea, en 1998 su número ascendió a más de 45.000 y con el control de más de 280.000 filiales repartidas en todo el mundo, y con datos algo más lejanos en el tiempo, de 1994, se recuerda que estas empresas "representaron alrededor de un tercio de la producción mundial y su comercio interior aproximadamente un tercio del comercio mundial"3. Desde un ámbito político de primera magnitud se nos recuerda que la mundialización no es sólo de carácter económico, aún cuando este aspecto sea el más relevante, ya que cada día cruzan fronteras internacionales " 500.000 pasajeros en transportes aéreos, 1.500 millones de correos electrónicos y 1,5 billones de dólares"4; y desde ámbitos intelectuales no menos importantes se enfatiza que el cambio más importante que implica la globalización respecto a etapas anteriores viene dado por la importancia que asumen los mercados financieros que operan en tiempo real, y que acompañados por la revolución de las comunicaciones y la extensión de la tecnología informática, posibilitan que "más de un billón de dólares se negocien al día en transacciones financieras societarias" 5 . Respecto a los cambios tecnológicos, uno de los autores más reconocidos profesionalmente por sus estudios en este ámbito, el profesor Manuel Castells, afirmaba recientemente al referirse a Internet que "no es simplemente una tecnología, sino que es la forma de organización de la nueva economía y de la nueva sociedad. Se puede decir que esta era sin Internet es como la era industrial sin electricidad"6; y también el gobernador del Banco de España L. A. Rojo ha señalado hace poco tiempo la importancia del cambio tecnológico y las diferencias de ritmo existentes entre los Estado Unidos y la Unión Europea en este terreno, al mismo tiempo que ha destacado la incertidumbre

3 Comunicación de la Comisión al Consejo, al Parlamento Europeo, al Comité de Regiones y al Comité Económico y Social. Competitividad de las empresas europeas frente a la mundialización. Cómo fomentarla. Bruselas, 20.01.99, COM (98) 718 final. El documento enfatiza además la importancia de los recursos intelectuales para toda empresa, ya que el valor de ésta "viene determinado más con respecto a su potencial intelectual y la calidad de gestión que con respecto a sus activos tradicionales" (pág.8).

4 Así se manifestaba el presidente norteamericano Bill Clinton en el discurso pronunciado el pasado 15 de junio de 1999 en la 87a Reunión de la Conferencia Internacional del Trabajo de la OIT, que hacía una llamada a encontrar una tercera vía "que nos permita incrementar al máximo el potencial del mercado y la justicia social, la competencia y el sentido de comunidad".

5 A. GIDDENS, La tercera vía. La renovación de la socialdemocracia. Madrid, Ed. Taurus, 1999.

6 Entrevista publicada en La Vanguardia, suplemento Economía y Negocios, 11 de marzo de 2000, pág. 4. 
existente sobre cómo se desarrollará este fenómeno en el futuro inmediato ${ }^{7}$.

Pero la globalización económica tiene su contrapunto en la creciente importancia que asume el ámbito local en múltiples decisiones, muchas de ellas decisivas en el ámbito de la creación de empleo, como se encargan de recordarnos los numerosos estudios efectuados por la OCDE y la Unión Europea sobre las iniciativas locales de empleo y los nuevos yacimientos de empleo; "localización" de muchas actividades que requiere un intenso grado de colaboración y cooperación entre los distintos poderes públicos y los diversos agentes sociales implicados para llevar a buen puerto los objetivos propuestos, en el bien entendido que ello requiere de nuevos marcos jurídicos y económicos que den cobertura suficiente a las medidas adoptadas en dichos ámbitos. Cómo conjugar correctamente el cambio global con las decisiones locales en el terreno económico y social es probablemente uno de los retos más significativos del futuro inmediato y, como ha subrayado muy recientemente el Banco Mundial en su Informe sobre desarrollo mundial para el período 1999-2000, "el éxito de un país en el s. XXI dependerá de su capacidad para manejar estas dos fuerzas de forma simultánea"8. Éxito que también dependerá de cómo conjugar la apertura a la nueva economía del conocimiento con el refuerzo de la protección social9.

7 Además de la revolución tecnológica en el ámbito de la información y de las comunicaciones, L.A. Rojo subraya dos elementos más que condicionan el devenir de la economía mundial: los desequilibrios en los pagos internacionales entre las principales áreas monetarias, "que han alcanzado niveles preocupantes", y "la fortísima revalorización de los activos bursátiles" y las dudas sobre su solidez o sobre su carácter de burbuja financiera. Cfr. "Reflexiones sobre la situación económica internacional". Conferencia pronunciada en el club Siglo XXI. Boletín Económico del Banco de España, abril 2000, págs. 1-11

8 En el Informe se subraya las nuevas oportunidades que genera la globalización como el más fácil acceso a los mercados y las transferencias de tecnología, pero también sus aspectos oscuros como son la inestabilidad y los cambios que genera, el temor a la pérdida de puestos de trabajo o la creciente dependencia de flujos irregulares de capitales importados; respecto a la localización se valora su importancia por las mejoras habidas en los sistemas de comunicación y transporte y supresión de barreras comerciales, y el hecho de que la actuación política puede ser más eficiente, aún cuando también se apunta el riesgo de conflictos económicos y sociales derivados de la exacerbación de la autonomía. Vid. Cinco Días, 16 de septiembre de 1999.

9 Para el profesor M. CASTELls hacer nueva economía implica "adoptar la flexibilidad geográfica, la flexibilidad del trabajo, la liberalización del mercado de capitales, la ruptura de los últimos elementos de monopolio de telecomunicaciones, el desarrollo de las estructuras de capital riesgo", y junto a ello "hay que reforzar el sistema de protección social para los ciudadanos, hay que reforzar el sistema de educación (políticas contra la exclusión y la marginación) e 
En definitiva, no parece que el debate que afecta al mundo del trabajo sobre el proceso de globalización económica y su impacto sobre las condiciones de vida y de trabajo de buena parte de la población pueda reducirse a una simple aceptación o rechazo del mismo, sino que las respuestas sociales deben tratar de darse a escala coordinada internacional y por medio de los foros internacionales y organizaciones sociales de dicho ámbito; y plantearse seriamente en este debate, y es un debate que fue abierto desde la década de los ochenta y aún sigue estándolo, si el problema al que se enfrentan buena parte de las personas no es la falta de trabajo sino la falta de ingresos, y que al mismo tiempo que hay que apostar por una redistribución del trabajo también hay que hacerlo, como subraya André Gorz, "por una distribución de riquezas para cuya producción el capital emplea un número cada vez más reducido de trabajadores"10. En el debate sobre qué medidas adoptar para garantizar un empleo y una renta adecuada para todos habrá que tener la habilidad suficiente para conjugar lo realizable con lo necesario, habrá que unir ambas realidades y asumir que algunas medidas que se propongan, que se están proponiendo, pueden recibir fuertes críticas y ser tildadas en sentido peyorativo de utópicas o irrealizables; pero, como recordaba hace varios meses en un discurso el secretario general de la Organización Internacional del Trabajo, Juan Somavía, "hay que saber que siempre que se proponga una idea nueva alguien va a decir que es imposible, y sabemos que lo que hoy parece imposible, mañana será posible. sabemos que hoy están ocurriendo cosas en el mundo que 30 años atrás parecían irrealizables, y sin embargo ahí están".

En este marco global, ¿cómo se sitúa la Europa comunitaria?; o dicho de otra forma, ¿cuáles son algunos de los retos más relevantes y algunas de las cuestiones más significativas que tiene que abordar en el futuro inmediato, en el nuevo milenio, para hacer efectiva la cohesión social que tanto se

impulsar políticas de representación de los ciudadanos a todos los niveles de la Unión Europea". Cfr. Entrevista..., ob. cit., pág. 5.

10 La tesis central de GoRz, que ha cambiado sus criterios en épocas recientes respecto a los defendidos con anterioridad, es que hay que garantizar un ingreso suficiente incondicional a toda persona, y que se trata de desconectar del trabajo "el derecho a tener derechos, y sobre todo el derecho a lo que es producible o producido sin trabajo, o cada vez con menos trabajo. Se trata de tomar nota que ni el derecho al ingreso, ni la ciudadanía plena, ni el desarrollo y la identidad de todos pueden estar centrados en la ocupación de un empleo y depender de ello. Y de cambiar la sociedad en consecuencia". Cfr. Miserias del presente, riqueza de lo posible. Barcelona, Ed. Paidos, 1998, pág. 91. 
predica en todos sus documentos oficiales desde la constitución de la Comunidad Económica Europea por el Tratado de Roma de 1957? Veámoslo someramente a continuación, pero antes subrayo que las políticas sociales europeas ${ }^{11}$ deberían contribuir a potenciar el crecimiento de los derechos sociales en otros países y continentes, sirviendo como ejemplo guía para que el desarrollo social acompañe al desarrollo económico a escala global, pues tal como ha dicho muy gráficamente el secretario general de la Confederación Europea de Sindicatos, Emilio Gabaglio, "Europa no puede ser una isla de felicidad en un mar tormentoso", y un reciente informe de la OIT sobre los derechos laborales en el mundo pone de manifiesto que esta "isla de felicidad", en la que ciertamente también hay un número no despreciable de náufragos desprotegidos, está rodeada de numerosos países en los que se acrecienta "la brecha de la representación en el mundo del trabajo" como consecuencia de la segmentación de los mercados laborales y de las reestructuraciones de la producción en el marco de una economía cada vez más internacionalizada12.

Europa tendrá que dar respuesta adecuada a los desafíos que tiene ante sí, tales como las nuevas tendencias demográficas y sociales, la mundialización, la revolución de la información y de la comunicación, y la nueva economía basada en el conocimiento. La potenciación del desarrollo tecnológico, la educación y la cohesión social son claves, según se constata en documentos comunitarios, para alcanzar la eficacia económica y lograr una mayor prosperidad para la ciudadanía.

En el ámbito más concreto de las relaciones de trabajo y de la protección social Europa también tiene ante sí retos importantes a los que debe responder, algunos de los cuales serán abordados con mayor detalle en las páginas siguientes ${ }^{13}$ y de los que ahora sólo quiero apuntar los aspectos más

11 En mi exposición utilizo una noción deliberadamente amplia de política social, que incluye no sólo las referencias a normativa laboral y de seguridad social sino también las medidas adoptadas por las instituciones europeas en los ámbitos de la política regional y de cohesión social por medio de la intervención de los fondos estructurales comunitarios.

12 OIT. Su voz en el trabajo. Ginebra, 2000. Un dato significativo es el crecimiento de la economía informal, que supone más del $80 \%$ de los nuevos empleos de América Latina y el $93 \%$ de los empleos de África en los años 90". Puede consultarse el texto en la página web de la OIT.

13 Vid. el artículo de Hedva Sarfati “La protección social en un nuevo mercado laboral: posibles repercusiones políticas", publicado en la Revista de la Asociación Internacional de Relaciones Trabajo, 2000. 
significativos: la problemática del desempleo, con sus secuelas de exclusión social; la creciente participación femenina en la vida laboral; el incremento del empleo atípico; los cambios en las estructuras laborales y los perfiles de empleo derivados del auge experimentado por la nueva economía; la reducción del período dedicado al trabajo a lo largo de la vida de una persona, ya que la tendencia es a retrasar la entrada y adelantar la salida del mercado de trabajo; los cambios en las estructuras familiares, con el aumento de las familias monoparentales y de las personas que viven solas; en fin, el aumento de la esperanza de vida y sus consecuencias sobre los sistemas de proteción social en términos de la necesidad de dedicar mayores cantidades a pagar las correspondientes pensiones.

\section{Reflexión general sobre la Europa social}

El estudio y análisis de la realidad europea ${ }^{14}$, de los retos y problemas que debe abordar la Europa comunitaria desde sus orígenes, ha estado presente en buena parte de las reflexiones, seminarios y publicaciones de Cij desde su creación, tanto por medio de aportaciones de la propia institución como por la de muchos miembros de ésta. Su tarea ha sido en buena medida, tal como señalaba al iniciarse la década de los noventa el presidente Ignasi Salvat, con ocasión de la presentación de una publicación, "colaborar al trabajo de reflexión y de acción que tantos cristianos y tantas personas con preocupación social y política están llevando a cabo para conseguir un futuro europeo entusiasmador y más solidario"15. En este artículo me propongo continuar en esta tarea, desde la afirmación previa de que defiendo una Europa que tenga un rostro social.

Pero, ¿qué es la Europa social? Decía en 1992, con ocasión de la aprobación del Tratado de Maastricht y creo que sigue siendo válida dicha tesis, que había de avanzarse en la reducción gradual y paulatina de las desigualdades regionales, económicas y sociales en el seno de la Comunidad; me apoyaba

14 Vid. un exhaustivo análisis del proceso de construcción europea, en sus distintas vertientes,en J.J. Romero y A. Rodero (directores). España en la Unión Europea. más allá del euro. Córdoba, Publicaciones ETEA, 1999. Coincido con la tesis defendida en la introducción del libro cuando se afirma que "la dinámica europea es obra humana, que ha de ser valorada en función del bienestar de la población, y no es indiferente cuál vaya siendo su orientación y su contenido"(pág. 2).

15 Europa. Posibilidades y dificultades para la solidaridad. Cij, Barcelona, 1991, pág. 5. 
en dictámenes del Comité Económico y Social para defender que el objetivo final no debe ser sólo el logro de una mejora absoluta de las condiciones económicas, sino que también debe lograrse un equilibrio real en términos de aproximación de los ingresos disponibles, el acercamiento de las estructuras económicas y sociales y de las relaciones laborales, de los niveles de bienestar y con una decidida implicación de los agentes sociales en todas las cuestiones que les afecten; en definitiva, avanzar en la cohesión económica y social, pero no sólo a escala comunitaria, sino también en el seno de cada Estado miembro, pues de poco serviría abrir caminos a la cohesión supraestatal si se mantienen o se incrementan las diferencias regionales.

1957 vio la aparición de la Comunidad Económica Europea, un intento claro y manifiesto de lograr la unión económica y política a medio plazo entre países que pocos años antes se habían visto cruelmente enfrentados durante la segunda guerra mundial. En dicho intento, cuyos resultados han sido altamente positivos en el aspecto económico pero mucho menos en el político y social hasta el momento presente, los Estados fueron conscientes de que debían trabajar de forma conjunta y coordinada, sin poder imponer unos sus pretensiones sobre otros; unanimidad, consenso y diálogo fueron y han sido durante muchos años las vías por las que ha avanzado el proceso de construcción europea y que deben merecer una valoración global positiva, pues tal como ha puesto de manifiesto el director académico de Cij, J osé Ignacio Gonzáles Faus, seguir esta vía ha implicado lentitud en todos los procesos, "pero ha sido también asombrosa la paciencia de todos para no abandonar el empeño, incluso en los momentos en que parecía definitivamente sin salida"16.

En el ámbito social cabe destacar que los avances a lo largo de toda la historia nunca se han producido de forma lineal y sin obstáculo alguno en el camino, y la construcción euro pea no escapa a esta constatación. Después de la aprobación de cada nuevo Tratado ha sido, y creo que sigue siendo, necesaria la presión del movimiento sindical y de las fuerzas políticas progresistas para llevar al convencimiento de las instancias comunitarias que difícilmente una gran parte de la población europea, los trabajadores, se sentirán implicados en la construcción del espacio común europeo si no advierten que las ventajas de su creación y el reconocimiento de derechos también se dirigen hacia ellos. En ese espacio común juega un papel muy importante la defensa y mantenimiento, ciertamente con todas las reformas

16 Ibidem, pág. 7. 
que sean necesarias, del Estado del Bienestar, con la potenciación de los aspectos sociales y para evitar que éstos queden relegados a un segundo plano y jueguen un mero papel secundario respecto a los aspectos económicos, financieros y monetarios. Porque el modelo social europeo se ha demostrado válido para abordar el proceso de construcción europea y debe seguir siendo un pilar de ésta; un modelo que tal como ha afirmado en numerosas ocasiones el que fuera presidente de la Comisión, J acques Delors, es una combinación del mercado y sus límites, con compromisos entre el capital y el trabajo, y con la intervención del Estado en ámbitos básicos como son, por ejemplo, la educación, la investigación y la ordenación del territorio.

Este modelo social que defiendo debe tratar de potenciar la creación de empleo y su estabilidad, la igualdad de oportunidades, y buscar la cohesión social, potenciando la lucha contra la exclusión social y dando más poder a las administraciones públicas más cercanas a los ciudadanos, es decir las regionales y locales, para que puedan intervenir con competencias propias en la elaboración de la política social europea. Coincido con las tesis del Comité Económico y Social y del Comité de las Regiones cuando afirman que el modelo social pasa igualmente por el reconocimiento de la estrecha interrelación de la política social con la política económica y en que es necesario que haya normas sociales mínimas que garanticen la cohesión social en la Comunidad, aplicables también a los nuevos Estados que se incorporen a la Unión Europea; asimismo coincido en que debe reforzarse la protección social y los servicios públicos, pues protección social suficiente y eficiencia económica se demuestran que van estrechamente unidos ${ }^{17}$, y además "la política social no son sólo normas laborales, sino que debe prestar especial atención a otras cuestiones como son las tendencias demográficas, la protección social o la lucha contra el racismo"18. Por fin, cabe destacar que en este modelo social cobrará cada vez más importancia la intervención de los agentes sociales, dado que el nuevo marco monetario a

17 Tesis también defendida en un documento relativamente reciente de la UGT. Construyamos la cara social del euro. Propuesta de UGT para la convergencia real. 16 de abril de 1999. El sindicato incluye entre sus propuestas el incremento del gasto estatal español en protección social hasta equipararnos a la media europea, reducir nuestra diferencia del PIB con la media europea, y “lograr que el salario mínimo interprofesional español sea como mínimo el $60 \%$ del salario medio neto (en la actualidad es del $42 \%$ ) siguiendo las recomendaciones contenidas en la Carta Social Europea".

18 Dictamen del Comité de las Regiones sobre la Comunicación de la Comisión: “Programa de acción social 1998-2000". DOCE C93, 6.4.1999, págs. 56-64. 
partir de la entrada en funcionamiento del euro lleva a que sindicatos y patronales deban ajustar las políticas negociadoras en materia salarial a esta nueva realidad y obligará probablemente a un esfuerzo importante de tales agentes para abordar las negociaciones a escala supraestatal.

Nos podemos preguntar ahora, en vísperas del nuevo milenio, cómo se plantea la Europa comunitaria su futuro. ¿Tendrá, tal como deseamos y defendemos en este artículo un rostro más social, con especial interés por las necesidades y preocupaciones de la mayoría de los ciudadanos, o prevalecerá un enfoque meramente economicista que coloca a la realidad económica en un pedestal y a la social en uno de los más bajos peldaños que conducen a ella?

La vía para avanzar en el camino deseado pasa tanto por el fortalecimiento de los instrumentos jurídicos en el nuevo Tratado de Amsterdam como por el incremento de la cohesión económica y social mediante los fondos estructurales y de cohesión.

Respecto al primero se han dado tímidos pasos para situar el ámbito social al mismo nivel que el económico aunque todavía queda mucho camino pendiente. La dimensión social comunitaria se ha reforzado por la incorporación al nuevo texto comunitario del Protocolo de Política Social anejo al Tratado de Maastricht y que no pudo incorporarse a éste ante la frontal oposición del gobierno conservador británico. Así mismo, se ha incorporado un título sobre empleo, en donde se reconoce a la Unión competencias en materia de coordinación de las políticas de empleo estatales; y, aunque se trata de mera coordinación de las políticas de empleo estatales, ya está suponiendo en la práctica un cierto avance, vía planes nacionales de empleo, en la articulación de las políticas de empleo de cada Estado con las de Unión Europea, tal como ha defendido la profesora M ${ }^{a}$ Emilia Casas ${ }^{19}$ y como se constata en las Recomendaciones efectuadas por el Consejo al amparo de las vía abierta por el art. 128, apartado 4, del Tratado de Amsterdam²0.

Ahora bien, hay que seguir avanzando para reforzar el flanco social de las normas comunitarias, y una vía adecuada puede ser la de ampliar las competencias de la Unión al conjunto de la regulación colectiva de las

19 Entrevista publicada en Gaceta Sindical, № 173, marzo 1999, págs. 35-37.

20 Cfr. "Recomendaciones del Consejo de 14 de febrero de 2000 sobre la aplicación de la política de empleo de los Estados miembros". DOCE L52, 25.2.2000, págs. 32 a 40. 
condiciones de trabajo (recordemos que ahora quedan fuera del ámbito comunitario competencias tales como la regulación del derecho de libertad sindical, huelga y cierre empresarial), incorporar el texto de la Carta comunitaria de derechos sociales fundamentales de los trabajadores al capítulo social del Tratado de Amsterdam para que se convierta "en norma jurídica comunitaria de carácter vinculante", y ampliar los ámbitos competenciales en los que se puedan adoptar decisiones por mayoría cualificada y no necesariamente por unanimidad ${ }^{21}$.

Respecto al fortalecimiento de la cohesión económica y social, el mantenimiento y consolidación de las partidas presupuestarias asignadas a los fondos estructurales y de cohesión para el período 2000-2006, un total de 213.000 millones de euros según los acuerdos adoptados en la Conferencia del mes de marzo de 1999 en Berlín de los J efes de Estado y de Gobierno, deberá servir para avanzar en la disminución de las diferencias entre los diversos territorios de la UE. Es especialmente importante destacar la reducción de los objetivos a los que se destinarán dichas partidas, que quedan ahora en tres: el $n=1$, que es la promoción del desarrollo y ajuste estructural de las regiones de desarrollo más lento y al que se acogerán básicamente las regiones cuyo producto interior bruto per cápita sea inferior al $75 \%$ de la media comunitaria; el no 2, consistente en la conversión económica y social de las zonas que se enfrentan a problemas estructurales, con especial atención a las zonas industriales y quedando cubierto como máximo el $18 \%$ de la población de la UE; el $n$ 우, por el que se prestará apoyo y atención a la adaptación y modernización de las políticas y sistemas de educación, formación y empleo.

Paso ya a señalar al gunas pistas que deben tomarse en consideración para abordar el estudio y potenciación del rostro social comunitario. En primer lugar, cabe destacar la importancia que están adquiriendo las políticas activas de mercado de trabajo, básicamente las de formación y orientación hacia los desempleados de larga duración, a fin de vincular estrechamente la percepción de una prestación económica con una actividad formativa o la prestación de un trabajo de utilidad social, y reduciendo el peso de las cantidades destinadas exclusivamente a cubrir la situación de desempleo; en términos positivos, esta política apuesta por la empleabilidad y la capacidad de adaptación de la población desempleada, y también de la que sufre riesgo

21 En esta línea de actuación se manifiesta G. SPyropoulos, "Les relations professionnelles dans le tourbillon de la mondialisation". Droit Social, mars 1999, págs. 230-238. 
de serlo, para reducir los índices de desempleo; pero desde una visión no tan bonita o idílica se puede y debe criticar que en ocasiones las medidas dirigidas a los desempleados no sean tanto una vía para facilitar su reincorporación al mercado de trabajo sino un elemento de control social ante una creciente demanda por buena parte de la sociedad de una "más eficaz" asignación de los recursos públicos, sin que existan reales posibilidades de retornar al mercado de trabajo.

De ahí que en un estudio realizado por las Iglesias inglesa e irlandesa se argumente que, sin cuestionar la necesidad de abordar medidas que tiendan a la reducción del desempleo, "las ayudas que se ofrecen son a menudo vistas por los desempleados como una medida de presión que dirige la sociedad contra ellos, para buscar empleos que no existen o para luchar por empleos que no tienen posibilidad alguna de alcanzar"22. También deberá intentarse que las medidas dirigidas a facilitar el acceso al empleo de los desempleados les permitan reincorporarse al mercado regular y no acabar siendo un círculo cerrado, un compartimento estanco, del que no pueden salir dichos desempleados.

En segundo lugar, debe mencionarse el impacto de los cambios demográficos. Mientras que a escala mundial sigue creciendo el número de habitantes del planeta, en los países desarrollados, y dentro de ellos el conjunto que forman la Europa comunitaria, sigue produciéndose una caída de la natalidad y una correlativa disminución del porcentaje que su población supone sobre el total mundial. Dato significativo es que al iniciarse 1998 sólo el 6' $4 \%$ de los alrededor de 5.886 .800 miles de personas que componían la población mundial, habitaba en la UE, y que su crecimiento desde 1996 a 1997 se ha producido no tanto por el incremento natural de la población (39\%) sino principalmente por el aumento de las migraciones $\left(61 \%{ }^{23}\right.$; en efecto, la incidencia de las migraciones en el crecimiento de la UE ha sido del $75 \%$ en 1995 y ha descendido ligeramente hasta el $62 \%$ en 1998, constatándose que países como Grecia, Italia y Suecia hubieran decrecido en población si no se hubiera producido el fenómeno migratorio.

22 Council of churches for Britain and Ireland (CCBI). Unemployment and the future of work. An enquiry for the churches. Londres, CCBI, 1997, pág. 22 (hay traducción española publicada por Sal Terrae en 1999)

23 "Premiers résultats de la collecte des données démographiques pour 1997 en Europe". Eurostat. Statistiques en bref. Population et conditions sociales. № 9/1998. 
En esta realidad europea cada vez habrá mayor número de ciudadanos que requerirán prestaciones de los sistemas públicos de protección social por haber alcanzado la edad legal o pactada de jubilación, dado que otro elemento relevante es el envejecimiento de la población europea. La tasa global de fecundidad de la UE ha pasado de 2'59 hijos en 1960 a 1'45 en 1998, mientras que en el mismo período ha pasado del 11 al $16 \%$ el número de personas mayores de 65 años, y dentro de diez años los paises comunitarios tendrán el doble de personas mayores de 65 años que en 1960 (69 millones frente a 34).

Datos aportado por la Comisión Europea subrayan los cambios que se producirán en el mercado laboral en los próximos años, y que cualquier política de empleo deberá tomar en consideración, por el descenso de la población joven y el incremento de la población madura y de la tercera edad laboral: entre 1995 y 2025 el número de jóvenes menores de 20 años disminuirá en nueve millones y medio, un $11 \%$ respecto del número actual; el grupo de 20 a 59 años disminuirá en más de 13 millones, un $6^{\prime} 4 \%$ respecto a la situación actual, mientras que los adultos de más de 60 años aumentarán en más de 37 millones, es decir en más de un $50 \%$ con relación a la realidad actual, debido al acceso de los "baby-boomers" de los años 50 y 60 a la edad de jubilación ${ }^{24}$. A título de ejemplo significativo, veamos la realidad alemana: en 1998 los mayores de 65 años representan el $16 \%$ de la población, mientras quese calcula que ese porcentaje será del $30 \%$ en el año 2040; o dicho en otros términos, "hoy 100 trabajadores financian a 35 pensionistas, mientras que en el 2040 la misma proporción de activos tendría a su cargo 48" 25.

En tercer lugar, cabe enfatizar la importancia de la formación, pues los resultados del empleo «tienden a estar cada vez más determinados por las capacitaciones y conocimientos técnicos que poseen los individuos» como ha destacado la OIT ${ }^{26}$; formación en especial para las personas desemplea-

24 Informe de la Comisión al Consejo y al Parlamento Europeo. La situación demográfica de la Unión Europea 1995. Bruselas, 23.02.96, COM (96) 60 final, págs. 4-5. Otro dato preocupante que apunta el estudio es que el envejecimiento de la población va unido al incremento de la precarización del empleo y al aumento del desempleo, situación que puede dificultad las condiciones de acceso a una pensión, "Io que podría implicar divisiones más importantes entre los futuros jubilados, o incluso entrañar la exclusión social de algunos de ellos" (pág. 27).

25 B. Stern, "Les effets du papy-boom". Le Monde, 26 de septiembre de 1998.

26 Vid. Oficina Internacional del Trabajo. Consejo de Administración. 271āa reunión, marzo de 
das, que les ha de servir no únicamente para acceder al mundo laboral sino que también ha de ayudarles a enfrentarse a las nuevas realidades que son producto de los cambios sociales y productivos. Pensando no sólo en ellos, sino en el conjunto de la población, creo que el auténtico desafio para los sistemas educativos en el futuro inmediato consistirá en crear las condiciones para ofrecer a todas las personas oportunidades de educación y formación a lo largo de toda su vida, pues cada vez será más patente «que hay un conjunto sin solución de continuidad que forman la educación, la formación inicial y la formación a la largo de toda la vida» ${ }^{27}$. Tanto el aprendizaje como la formación son procesos contínuos, y de ahí que la adquisición de aptitudes fundamentales como la lectura, la escritura y el cálculo formen la base de toda iniciativa posterior en materia de formación, y que "el hecho de aprender a aprender es considerado como un elemento clave del aprendizaje, de la educación y de la formación"28.

Conviene destacar que la reducción de vida media de los conocimientos y la mayor esperanza de vida influyen de forma decisiva en los cambios que ya se están produciendo tanto en el mundo de la educación como en el de la formación, ya que si en el pasado "Ios conocimientos que una persona adquiría sobre su trabajo cambiaban muy poco mientras estaba en activo en el mercado laboral, ahora se enfrenta a una situación en la que necesita una formación constante para estar al día de los últimos avances"29. En un

1998. Documentos publicados en la web de la OIT. Más recientemente, vid. La formación para el empleo: la inserción social, la productividad y el empleo de los jóvenes. Conferencia Internacional del Trabajo, 88a reunión, Ginebra, junio 2000.

27 Ibidem. A destacar que la OIT se muestra especialmente sensible a cómo garantizar que las normas reguladoras de las políticas de formación "constituyan un mecanismo eficaz para la incorporación e integración de los grupos vulnerables en el empleo" y que eviten convertirse en un mecanismo que "exacerbe las desigualdades que pueden existir en el acceso a la formación y al empleo".

28 C. et B. MC NALLy, "La formation/l'apprentissage et les nouvelles formes d'organisation du travail face aux nouvelles technologies de l'information et la communication". Travail thématique européen ADAPT, septembre 1999. Los autores defienden la necesidad de que el proceso formativo sea gradable para las personas y que debe hacerse del aprendizaje un placer, y apuestan por "el aumento de la flexibilidad (personalización) de las formaciones, ya sean impartidas por las empresas, o bien por los servicios de educación, de formación y de ayuda social".

29 O. Gianini y P. M. LiedtKe, El dilema del empleo. El futuro del trabajo. Barcelona, Ed. Círculo de Lectores, 1999, pág. 124. Los autores formulan una propuesta ciertamente novedosa pero también muy difícil de llevar a la práctica: "en algunos casos se podría plantear que 
documento elaborado por la UNESCO se subraya acertadamente la cada vez más estrecha relación entre la enseñanza superior y el mundo del trabajo, desde una relación de complementariedad y no de subordinación de la primera al segundo: "la interacción y no una simple adaptación sistemática de la enseñanza superior, el partenariado, es decir la relación entre iguales y no la subordinación al mercado debe caracterizar las relaciones entre la enseñanza superior y el mundo del trabajo"30.

En cuarto lugar hay que apuntar la existencia de un amplio debate, que ya en su momento lanzó a escala internacional la OIT, sobre cómo combinar adecuadamente la flexibilidad y la seguridad laboral, muy centrado el debate en el seno de la UE en el ámbito de la utilización de las modalidades contractuales de duración determinadas o atípicas, que suponen en la actualidad alrededor del $13 \%$ de la contratación (subiendo a poco menos del $32 \%$ en España), predicándose la necesidad de conseguir que el trabajador mejore permanentemente sus cualificaciones profesionales para poder así mantenerse en el mercado de trabajo y no simplemente en el mismo empleo, al tiempo que se evita la utilización artificial de las contrataciones atípicas y se garantiza una protección social adecuada a los trabajadores que presten sus servicios bajo alguna de las situaciones, cada vez más frecuentes, diferentes del empleo a tiempo indefinido y a jornada completa. Afortunadamente se va abriendo paso en sedes internacionales políticas y sociales la necesidad de restablecer unas reglas del juego que combinen adecuadamente flexibilidad, seguridad y respeto de los derechos fundamentales de la persona en toda relación laboral, concluyendo poco a poco lo que gráficamente se dio en llamar "la época de las ingenuidades liberalizadoras en el terreno laboral"31, basadas en las tesis de que bastaría con desmantelar las legislaciones laborales nacionales para que la economía mundial funcionara correcta y perfectamente.

En este debate, también cobra toda su importancia cómo se organiza el

determinadas titulaciones pierdan automáticamente su validez a menos que sean refrendadas por nuevos exámenes o por la evidencia de que los conocimientos técnicos se mantienen al día" (pág. 265).

30 Vers un agenda 21 pour l'enseignement supérieur. Documento de trabajo preparado para la Conferencia Mundial celebrada en octubre del 5 al 9 de octubre de 1998, pág. 9.

31 A. SupIOT, "Introducción a las reflexiones sobre el trabajo". Revista Internacional del Trabajo. Vol. 115, 1996/6 (número especial: "Reflexiones cruzadas sobre el trabajo y su porvenir"), pág. 658. 
trabajo y cuál es el grado de participación de las personas que trabajan en dicha organización, a fin de tener más voz en las decisiones que les afectan y poder aspirar a un mayor equlibrio entre la vida familiar y la laboral, y de ahí que se enfatice en documentos internacionales que la flexibilidad no deba considerarse como un fin en sí misma, sino que debería servir "tanto para mejorar la competitividad y la productividad de las empresas como para mejorar la calidad de vida y las condiciones de trabajo"32. En modo alguno sería aceptable una flexibilidad laboral que tuviera como razón de ser una mayor inseguridad de las personas que trabajan y una mayor precariedad en el mercado laboral, y que sólo facilitara la protección de los trabajadores altamente cualificados.

\section{El marco social europeo}

Para efectuar propuestas de cambio, para abordar una Europa de rostro más social en donde se ponga el acento en la creación de empleo de calidad, en un crecimiento económico sostenible y en la mejora del potencial de innovación y creatividad, debemos conocer cual es el "mapa social" de la UE. Con carácter previo señalo que entre el conjunto de propuestas y recomendaciones adoptados en los últimos tres años con ocasión de las directrices de política de empleo aprobadas por el Consejo cabe resaltar por su importancia en relación con la materia objeto de este trabajo, tal como he apuntado con anterioridad, la potenciación del aprendizaje a lo largo de toda la vida laboral, la creación de empleo en el sector de servicios, donde no hay duda que se están generando la mayor parte de nuevos empleos, nuevas actividades y nuevas ocupaciones (con especial atención a las posibilidades abiertas en el sector del turismo), la modernización de la organización del trabajo con una apuesta por las relaciones de partenariado, y la lucha contras las desigualdades por razón de sexo y la adopción de medidas transversales que

32 Cfr. "Le futur du travail. Le travail du futur". Travail Thématique européen ADAPT. Nouvelles formes d'organisation du travail et société de l'information. Document de synthèse, septembre 1999. El documento subraya que hay tres ejes de trabajo en todo lo que se refiere a la nueva organización del trabajo: "la flexibilidad en la producción, en la organización, en el tiempo de trabajo, en las relaciones contractuales, incluyéndose aquí el teletrabajo y el trabajo a distancia; la cooperación y la puesta en red de las organizaciones y las ventajas que ello engendra para las empresas; las nuevas respuestas en términos de formación, tendentes a preparar a los trabajadores y las empresas a hacer frente a la modernización de la organización del trabajo". 
potencien la igualdad y que tiendan a incrementar el número de parejas que tenga una fuente de ingresos familiar dual ${ }^{33}$.

En definitiva, se trata de adoptar medidas que conviertan en realidades tangibles las grandes afirmaciones habitualmente contenidas en las declaraciones de los Consejos Europeos, siendo un ejemplo claro el celebrado en Colonia el 3 y 4 de junio de 1999, en el que se acordó potenciar el fomento activo y preventivo del empleo, y se defendió la existencia de unas normas mínimas sociales a escala europea, tratando de conjugar y lograr "una unión armoniosa entre competitividad, flexibilidad y protección social de los trabajadores" 34 .

Los aspectos de ese "mapa social" que considero más relevantes para conocer, estudiar y analizar ${ }^{35}$, son los siguientes:

A) En 1999 la UE ocupaba a 155'3 millones de personas, con una tasa de ocupación del $62 \%$, y una diferencia a la baja de cerca de 13 puntos respecto a la de EE.UU. Las diferencias se centran básicamente en los grupos de jóvenes de 15 a 24 años, las mujeres de edades intermedias y las personas de la tercera edad laboral entre los 55 y 64 años $^{36}$.

Respecto a las diferencias entre participación masculina y femenina en el

33 Las desigualdades no sólo se dan entre hombres y mujeres, sino también dentro del mercado de trabajo femenino, poniendo de manifiesto según la Comisión nuevas divisiones y nuevas diferencias de oportunidades entre "Ias mujeres con educación superior por un lado, y las mujeres con cualificaciones inferiores, por otra". Cfr. Informe anual de la Comisión. Igualdad de oportunidades entre mujeres y hombres en la Unión Europea 1998. Bruselas, 5.03.99. COM (1999) 106 final.

34 Con lenguaje más claro y directo, la Comisión ha defendido en otras ocasiones la potenciación de políticas de trabajo activas para prevenir el desempleo a largo plazo, que deberían ir acompañadas de "una reducción de los incentivos de los desempleados para seguir acogiéndose al subsidio por desempleo". Cfr. Informe de la Comisión. Reforma económica y estructural en la UE (Cardiff II). Bruselas, 17.02.99, COM (1999) 61 final.

35 Cfr. A. Franco, "Enquête sur les forces de travail. Principal résultats 1999". Statistiques en bref. Population et conditions sociales. Thème 3- 5/2000.

36 Vid. una detallada comparación de los datos de EE.UU y de la zona euro en "Evolución y características estructurales de los mercados de trabajo de la zona del euro". Boletín Mensual del Banco Central Europeo, mayo 2000, págs. 61-78. En el informe se constata que "Ios problemas que afectan al mercado de trabajo parecen concentrarse en ciertos grupos sociales como las mujeres, los trabajadores más jóvenes y de más edad y los menos cualificados, mientras que la situación de los varones de 30 a 59 años parece comparable en términos generales entre la zona del euro y de Estados Unidos"(pág. 61). 
mercado laboral, la diferencia en la tasa de ocupación a favor de los varones se ha reducido desde 26 puntos en 1990 a 19 en 1997 (71'6 y 52'6 \%, respectivamente). La importancia de la participación de la mujer en el mercado laboral europeo debe medirse no sólo por el número de puestos de trabajo que ocupan, sino también tomando en consideración que "una cifra más elevada de empleo femenino crea puestos de trabajo para atender necesidades que antes no se retribuían, como el cuidado de los niños o de las personas mayores" ${ }^{37}$. Si comparamos los datos de la UE con los españoles veremos que existe una diferencia negativa por nuestra parte en la tasa de empleo femenina, ya quela media comunitaria es del 39' $9 \%$, con tasas del $50^{\prime} 1$ \% y 55 '2 \% en Reino Unido y Dinamarca, mientras que la española en el cuarto trimestre de 1999 era del $26^{\prime} 4 \%{ }^{38}$.

Con relación a las personas mayores de 55 años, es preocupante que la tasa de empleo haya descendido ocho puntos en la UE desde 1985, siendo en 1998 del 46 ' $5 \%$, destacando la caída masculina y el ligero aumento femenino. En foros comunitarios se plantea, y creo que acertadamente, cambiar la tendencia actual de concentración de la vida laboral entre los 25 y los 55 años de edad, por las dificultades que dicha concentración plantea tanto para conciliar la vida familiar y la laboral como por el coste económico que implica para el erario público debido a la mejora sustancial en la expectativa de vida de la población ${ }^{39}$, proponiéndose en las recomendaciones generales sobre el empleo en 1999 y 2000 desincentivar la jubilación anticipada por el coste económico que tal medida tiene para la colectividad. Según la UE la caída masculina es debida al fenómeno de las reestructuraciones industriales, y en muchas ocasiones no es voluntaria, habiendo puesto de manifiesto una encuesta del Eurobarómetro queal menos el $40 \%$ de los prejubilados hubiera

37 Comisión de las Comunidades Europeas. Informe sobre la tasa de empleo de 1998. Evolución del empleo en los Estados miembros. Bruselas, 14.10 .98 (1998) 572 final, pág. 14.

38 Vid. Secretaria Confederal de la Mujer CC.OO. La situación de las mujeres en el mercado laboral. Marzo 2000. Con datos de la EPA del 4ㅇtrimestre de 1999 se subraya que la tasa de actividad femenina es casi la mitad que la masculina ( $39^{\prime} 1$ y $66^{\prime} 3 \%$ ), y lo mismo ocurre con la tasa de empleo ( $30^{\prime} 4$ y $56^{\prime} 5 \%$ ), mientras que la tasa de paro femenina es más del doble que la masculina ( $22^{\prime} 4$ y $10^{\prime} 8 \%$ ). En relación con los contratos formalizados en el INEM en 1999, 13.242.531, el $41 \%$ correspondió a mujeres y el $51 \%$ a hombres, siendo los das modalidades más utilizadas para contratar a mujeres las de eventualidad (35'04\%) y a tiempo parcial ( $\left.28^{\prime} 01 \%\right)$ mientras que para hombre se utiliza más el contrato para obra 0 servicio (38'42\%), seguido de cerca por el eventual (35'76\%).

39 Commission Européenne. L'offre future de main d'oeuvre en Europe. Luxemburgo, 1999. 
preferido continuar ejerciendo una actividad profesional, y siendo cada vez mayor el número de personas entre 55 y 60 años con buena salud y que desean conservar alguna forma de trabajo o de implicación en la vida laboral más allá del fin oficial de su vida profesional.

B) El aumento del empleo, en 1999 con respecto al año anterior debe subrayarse, pues se ha producido un crecimiento neto de 3.200 .000 y con incremento del trabajo cualificado; ha habido un incremento de la actividad a tiempo parcial (el $80 \%$ de esta corre a cargo de las mujeres), y además se produce un aumento importante de la contratación temporal que alcanza ya el $13 \%$. La reducción global del número de horas trabajadas se debe a ese incremento de la actividad a tiempo parcial, si bien ha sido menor de la que porcentualmente correspondería, ya que ha ido acompañada de "un aumento del número medio de horas trabajadas por los trabajadores a tiempo completo" 40 .

C) Según datos de 1997, cerca del $47 \%$ de los desempleados de ambos sexos carecían de titulación salvo la escolaridad obligatoria. La importancia de garantizar una mejor formación escolar y de un reciclaje y formación permanente para las personas que están trabajando o desean trabajar es cada vez mayor. Es importante disponer de estudios y, cuanto más elevados en titulación, mejor, para acceder al mundo laboral. En la UE el desempleo de los titulados superiores es del $6 \%$ en 1997, frente al $10 \%$ de aquellos que habían finalizado los estudios secundarios y el $14 \%$ para quienes se habían detenido en el período de escolaridad obligatoria.

La inversión en la mano de obra actual es necesaria se si se observa que en la próxima década y a partir de los datos demográficos disponibles, "habrá tres millones menos de entradas en el mundo laboral y el número de personas mayores de 50 años aumentará en 9'6 millones" 41 . Fomento de la formación que pasa también por invertir en la potenciación de la actividad empresarial, creando desde ámbitos educativos una formación que posibilite para ello y que anime a la asunción de riesgos.

D) Debemos prestar especial atención a los jóvenes en la UE ${ }^{42}$, y en especial al núcleo duro de quienes encuentran más dificultades para inte-

40 Comisión Europea. El empleo en Europa 1998, Bruselas 20.11.98. COM (1998) 666 final, pág. 9.

41 Informe presentado por la Comisión dirigido al Consejo Europeo. Oportunidades de empleo en la Sociedad de la Información. Bruselas, 25.11.1998 COM (1998) 590 final, pág. 18.

42 Para analizar correctamente la problemática juvenil debe dividirse a la población joven en 
grarse en los dispositivos de inserción existentes en la mayor parte de países comunitarios, tratando de evitar efectos no deseados cuales son que se beneficie de las medidas de política de empleo un sector de la población juvenil que en cualquier caso hubiera podido acceder al mercado de trabajo. Así lo subraya también la OIT en sus documentos sobre la juventud cuando enfatiza con claridad que conviene concentrar los esfuerzos sobre los grupos de jóvenes "que tropiezan con especiales dificultades para obtener trabajo ${ }^{43}$, por ejemplo aquellos que terminan la escolaridad con calificaciones insuficientes o sin calificaciones, las minorías étnicas, los incapacitados y los que residen en zonas de elevado desempleo", sin olvidar tampoco "un restablecimiento del equilibrio en lo que respecta a las posibilidades de encontrar empleo de los hombres y mujeres jóvenes"44.

Con datos de 1999, había 45.862.000 millones de jóvenes de 15 a 24 años en la UE ${ }^{45}$ y cerca de cuatro de ellos se encontraban desempleados, con tasas de desempleo del 18 ' $3 \%$ frente al $9 \%$ de los comprendidos entre 25 y 49 años (a excepción de Alemania y Austria donde las tasas de ambos grupos son parejas a la baja), y con un incremento hasta el $28 \%$ para los recién salidos del mundo educativo, situación que demuestra según los informes comunitarios que "el primer año tras los estudios representa una fase crítica de la transición entre la escuela y el trabajo"46. Con datos empíricos se demuestra

cuatro grupos: "desempleado; inactivo (que no tiene ni busca activamente trabajo); en proceso de educación/formación (estudiante a tiempo completo); ocupado". Cfr. Commission Européenne. L'insertion des jeunes sur le marché du travail._Luxemburgo, 1999.

43 Entre las medidas adoptadas en sede comunitaria para luchar contra el desempleo cumplió un papel relevante el programa Y outhstart, a fin de dar respuesta a necesidades de colectivos desfavorecidos como jóvenes de regiones en declive, inmigrantes, las jóvenes, y los jóvenes que han abandonado los estudios.

44 N. O'HigGins, "El desafio de la ocupación de los jóvenes". Revista Internacional de la Seguridad Social, no 4, octubre-diciembre 1997, pág. 87.

45 La previsión es de pérdida de importancia del empleo juvenil numéricamente hablando, ya que se calcula que en el año 2005 habrá cerca de un millón menos de jóvenes de 15 a 19 años, y cerca de nueve millones menos de jóvenes entre 20 y 29. Cfr. Commission Européenne. L'insertion..., ob. cit.

46 Si se utiliza el dato del porcentaje de desempleo de jóvenes sobre el conjunto total de la población de dicha edad, tesis no desdeñable dado el elevado número de jóvenes inactivos por seguir estudiando una vez finalizada la escolaridad obligatoria, el porcentaje disminuye y va desde el $4 \%$ de Luxemburgo al $20 \%$ de Finlandia. "De l'école à la vie active. Faits marquants sur le chômage des jeunes". Eurostat. Statistiques en bref. Population et conditions sociales, no 13/1998. 
que el nivel de formación y la mayor titulación académica influyen de forma positiva en la posibilidad de acceder a un empleo, y de ahí que la mayor parte de países hayan introducido importantes reformas en los sistemas educativos en los últimos años, tanto para mejorar los niveles de escolarización como para reducir el número de jóvenes que abandonan los centros educativos sin haber obtenido titulación. Es preocupante que cerca de un $20 \%$ de jóvenes, según constatan estudios comunitarios, salgan de la escuela sin poseer los conocimientos y las capacidades suficientes para la economía actual, y de ahí que se trate de reforzar la formación profesional y de poner en marcha recorridos específicos de inserción para los jóvenes desfavorecidos o marginados y que hagan posible su incorporación posterior al mercado regular ${ }^{47}$. Los datos utilizados por el Banco Central Europeo refuerzan la tesis de que cuanto más bajo es el nivel de instrucción escolar más alta es la tasa de desempleo, ya que "más del $22 \%$ de los desempleados se encuadraban en la categoría de menos cualificados (es decir parado cuya ocupación anterior eras obrero no especializado), mientras que esta categoría representa sólo el 9'3\% del empleo total"48.

E) Hay que mejorar el contexto institucional del empleo, porque los datos comunitarios demuestran que no son los salarios los responsables de la poca creación de empleo en comparación con la realidad norteamericana. Los costes laborales por asalariado aumentaron en el período 94-97 sólo en un $50 \%$ de la tasa de crecimiento de la productividad en el trabajo, y de ahí que la participación salarial esté sólo ligeramente por encima del $60 \%$ del PIB en la UE, mientras que en EE.UU es del $66 \%$ y en J apón del 67 \%; por otro lado, los propios documentos comunitarios destacan que la regulación de la fiscalidad no ha sido precisamente favorable al empleo, ya que «entre $1980 \mathrm{y}$ 1996 la carga media de los impuestos y cargas sobre el trabajo en la UE ha aumentado de manera constante (de 35 a $43 \%$ ), mientras que para otros factores de producción ha disminuido, sobre todo para el capital (de 42 a 36

47 Con carácter general, la Comisión europea constata que un nivel adecuado de educación y formación no es suficiente por sí solo para lograr una adecuada integración en el mercado de trabajo, sino que es conveniente también que la formación este relacionada con el ejercicio de una actividad profesional, y de ahí que se defienda que "el hecho de que una parte significativa de la formación se realice en situación de trabajo proporciona al joven una cierta ventaja, en especial en el primer estadio de su integración en el mercado de trabajo". Cfr. L'insertion..., ob. cit.

48 Evolución y..., ob. cit., pág. 73. 
$\%)$ y ha permanecido estable para el consumo (14 \% ${ }^{49}$.

F) Cada vez más se pone de manifiesto el importante papel que deben jugar las autoridades regionales y locales en el diseño, elaboración y puesta en práctica de las políticas de empleo, en estrecha relación con los agentes sociales y con las organizaciones de la economía social, a fin de dar adecuadas respuestas a las necesidades sociales y medioambientales; en especial, el sector de la economía social, con su potencial de generación de empleo en ámbitos relacionados directamente con las necesidades de atención a las personas y con especial incidencia en el ámbito local, es uno de los que la UE considera que debe ser objeto de mayor atención, a fin de incrementar su cuota de participación tanto en el empleo global (en la actualidad alrededor del $5 \%$ ), como en el número de empresas implicadas (alrededor del $6 \%$ en el sector privado). Ahora bien, la llamada a la participación de las autoridades, fuerzas políticas y sociales locales y regionales, no debe hacernos olvidar la importancia de mantener un sistema público de empleo que garantice a escala estatal el principio de igualdad de trato entre todos los ciudadanos y que evite cualquier tipo de discriminación. En el diseño de esta nueva política de empleo debe concederse especial atención a los pactos territoriales para el empleo, por su enfoque cooperativo y porque implica a todos los agentes económicos y sociales en la resolución de los problemas planteados, creando un protagonismo directo de todos los sujetos implicados y abriendo nuevos campos de actuación para las políticas activas de empleo, así como obligando a los agentes sociales a "abrirse mentalmente" para buscar nuevas respuestas a los nuevos problemas ${ }^{50}$. Las iniciativas locales pueden ser sin duda un terreno adecuado en especial para que los jóvenes se incorporen al mundo del trabajo en ámbitos como la cultura, donde la Comisión Europea calcula que se encuentra ya cerca de un $2 \%$ de los empleos de la UE, espectáculos, deportes, acción social o entorno rural y urbano, por citar sólo algunos ejemplos significativos, ámbito profesionales en donde es necesario potenciar las acciones formativas. Tal como se enfatiza por el Comité de las Regiones es necesario potenciar el papel de las

49 Comisión de las Comunidades Europeas. Informe sobre..., ob. cit., págs 19-20.

50 “El empresario se acostumbra a desenvolverse no sólo en la lógica natural del interés de la empresa, sino en un interés más amplio del desarrollo de la zona. El sindicato se responsabiliza participando en decisiones que sin duda presentan características de opciones empresariales y de lógica costes / beneficios". Dictamen del Comité Económico y Social. «Los pactos terrritoriales para el empleo". DOCE C407, 28.12.98, pág. 71. 
autoridades locales y regionales en la elaboración de los planes nacionales de empleo, y en esta línea, "la cooperación entre las autoridades locales y regionales y los servicios públicos de empleo es igualmente necesaria a la hora de elaborar medidas políticas activas de fomento del empleo; determinar y aplicar planes para la inserción y la orientación profesional, y para organizar servicios de asistencia y apoyo a las iniciativas de desarrollo local que tengan como prioridad repercutir positivamente en el empleo"51.

G) ¿Más progreso, más desigualdades? Tal situación también se produce en la UE, donde se pueden observar grandes desigualdades entre los diferentes estratos de la población, aun cuando dicha desigualdad sea bastante inferior a la de regiones y Estados donde no existen o están poco desarrollados los sistemas de protección social. Si se analiza la distribución de la renta en trece Estados comunitarios de los quince que componen la UE (exceptuando Suecia y Finlandia) se comprueba que el $10 \%$ de la población con menor nivel sólo recibe el 2'6\% de la renta comunitaria, mientras que el otro $10 \%$ dela población con mayor nivel recibecerca del $24 \%$. Si a dicho dato le añadimos el de que en buena parte de los hogares del $10 \%$ de nivel de ingresos inferior hay trabajadores desempleados podemos llegar a la conclusión de que las desigualdades pueden ir creciendo de forma gradual y paulatina, en una sociedad donde el trabajo (asalariado o independiente) sigue siendo la fuente principal de ingresos, ya que como ponen de relieve estudios comunitarios el $70 \%$ de las rentas de los hogares europeos provienen de dicho trabajo, el $25 \%$ de las pensiones y otras transferencias a cargo de los poderes públicos, y el $5 \%$ restante del capital y otras fuentes de ingresos ${ }^{52}$. No obstante, conviene subrayar que la noción convencional de trabajo de nuestras sociedades desarrolladas abarca sólo a una minoría de trabajadores a escala mundial tal como recordaba el secretario general de la OIT Juan Somavía ${ }^{53}$, y además que la valoración del trabajo como la actividad más importante en la vida de la persona varía considerablemente en los

51 Dictamen de 18 de noviembre de 1999 sobre la "Propuesta de Directrices para la política de empleo de los Estados miembros en el año 2000". DOCE C57, 29.2.2000.

52 "Analyse de la répartition du revenu dans treize États membres de l'UE". Eurostat. Statistiques en Bref. Population et conditions sociales, no 11/1998.

53 Somavía destaca el carácter complejo del concepto de trabajo y manifiesta que este engloba "todas las actividades encaminadas a cubrir las necesidades vitales y a garantizar la supervivencia y el bienestar de la familia". "El futuro del empleo". El País, suplemento "World Media", 21 de abril de 1999, pág. 6. 
diversos Estados en razón de factores culturales, sociales y de bienestar social ya alcanzado ${ }^{54}$.

H) ¿Qué decir, respecto a los niveles de protección social en la UE? LOS últimos datos disponibles de 1998 ponen de manifiesto que el porcentaje de PIB dedicado a ésta alcanza el 28'2 \% como media comunitaria, habiendo descendido cinco décimas respecto al año anterior, si bien si compara con el dato de 1990 (25'4\%) se constata que se ha incrementado en casi tres puntos. Consecuencia lógica de la crisis de la primera mitad de los noventa, el mayor incremento de las partidas económicas en la primera mitad de esta década se ha debido a la protección por desempleo, medidas contra la exclusión social y medidas de apoyo a una política adecuada de vivienda. Por otra parte, la reducción operada desde 1993 en la media comunitaria dedicada a protección social, año en que se alcanzó el 28 '9 \%, se debe en gran medida a políticas restrictivas del gasto social aplicadas por diversas Administraciones Estatales ${ }^{55}$. En la actualidad, y sólo cito las cifras más significativas, la protección por vejez se lleva el 45'2 \% del total (es decir, el 12'2 \% del PIB), las prestaciones por enfermedad significan el $35^{\prime} 4 \%$, el $8^{\prime} 3$ va dedicado a la protección a la familia y el 7'5 \% para protección por desempleo ${ }^{56}$.

Un último apunte en esta materia. La política de protección social, por lo que respecta a la familia, debe tomar en consideración en toda Europa, aunque con diversos grados de intensidad en cada país, el incremento de familias monoparentales que en trece países comunitarios (exceptuando Dinamarca y Suecia) suponían ya cerca de siete millones en 1996, o lo que es lo mismo un $14 \%$ del total de familias con hijos a su cargo y siendo en un $84 \%$ una mujer la que está al frente ${ }^{57}$.

I) Debe constatarse la preocupación comunitaria por el incremento de las

54 Vid. "Actitudes hacia el trabajo". Boletín del CIS nำ23, enero-marzo 2000.

55 “La protection sociale dans I'Union Européenne". Eurostat. Statistiques en Bref. Population et conditions sociales, no 14/1998.

56 "La protection sociale en Europe". Statistiques en bref. Population et conditions sociales. Thème 3-2/2000.

57 "Les familles monoparentales: un phénomène qui prend de l'ampleur". Eurostat. Statistiques en bref. Population et conditions sociales, no 12/1998. El informe subraya que "el incremento del número de estos padres sin pareja es uno de los datos más relevantes de la evolución demográfica y social de los últimos años, y entre 1983 y 1996 su número ha aumentado en media del $55 \%$ en ocho paises de los que se dispone la información". 
nuevas formas de pobreza y exclusión social en el seno de la UE, situación queha llevado al Comité Económico y Social a lanzar "un grito dealarma" ante una situación en que la pobreza se extiende y agrava, y a la Comisión Europea a alertar ante las nuevas exclusiones que podrían aparecer en la nueva economía y sociedad del conocimiento, ya que puede crear diferencias relevantes entre "quienes poseen las competencias, las calificaciones y las aptitudes requeridas, y quienes carecen de ellas" $" 58$, además de manifestar su preocupación por el coste económico que supone el desempleo, la pobreza y la exclusión social, que se calcula que podría situarse entre el 12 y el $20 \%$ del PIB ${ }^{59}$. El CES afirma que el desempleo tiene un impacto especialmente fuerte entre los factores de pobreza, si bien se subraya igualmente que no siemprees la causa original y que "no todos los parad os son pobres, al tiempo que hay pobres -y cada vez son más numerosos- entre las personas que tienen empleos poco o nada cualificados, y por tanto con ingresos escasos y a menudo precarios" ${ }^{\prime 60}$. Esta afirmación se confirma empíricamente con los datos facilitados por un estudio de Eurostat sobre las rentas de los hogares de los países de la UE en 1993, y en el que se pone de manifiesto que la realización de una actividad profesional, ya sea por cuenta propia o ajena, no impide en muchas ocasiones que rentas derivadas del trabajo sean escasas en bastantes hogares, afirmándose que "cerca de un $53 \%$ de los ciudadanos dela UE que perciben una renta escasa viven en hogares que tienen una cierta actividad profesional. Según los países, de uno a dos tercios de la población de renta escasa forman parte de hogares en que uno o varios miembros ejercen una actividad asalariada 0 independienteduranteal menos un año"61.

58 "Construirel'Europe de l'inclusion". Comunicación presentada como aportación a la cumbre europea extraordinaria de Lisboa de los días 23 y 24 de marzo de 2000.

59 Cfr. Le Conseil Européen de Lisbonne. Un agenda de renouveau économique et social pour l'Europe. Contribution de la Commission Européenne au Conseil européen spécial de Lisbonne des 23 et 24 mars 2000, pág. 8.

60 "Dictamen sobre el coste de la pobreza y la exclusión social en Europa”. DOCE C284, 14.09.98, págs. 25 a 39. El texto fue aprobado en sesión plenaria del 1 y 2 de julio, por 130 votos a favor, 6 en contra y 14 abstenciones. La importancia de determinar el coste de la pobreza y exclusión viene dada según el CES por el hecho de que de una parte hay que concretar el coste de las medidas de tratamiento y de prevención, y de otra "cuál es la incidencia del déficit de consumo de los pobres y excluídos sobre la producción, el empleo, los ingresos fiscales y las cotizaciones sociales" (pág. 32).

61 "Faibles rémunerations et faibles revenus: une analyse UE-12 au niveau des ménages". Statistiques en bref. Population et conditions sociales. № 6/1998. Según el estudio "Ias rentas obtenidas por un empleo temporal, a tiempo parcial o por una actividad independiente, no 
Según muy recientes datos comunitarios, cerca del $12 \%$ de las personas que tienen un empleo viven en situación de pobreza, explicándose dicha situación "porque los trabajadores de hogares con rentas bajas ocupan a menudo empleos débilmente remunerados"62.

En el ámbito europeo la mayoría de los nuevos pobres proviene del grupo de trabajadores en paro, y dentro de ellos de los de larga duración, si bien hay que prestar atención cada vez más a nuevas realidades del mundo del trabajo que llevan a muchas personas a pasar de una situación de trabajo estable a otra de desempleo imprevisto y posterior exclusión social; de esta forma, no es infrecuente hoy en día referirse a "pobres de clase media", pues las organizaciones que prestan atención social reciben cada vez más "a un porcentaje importante de gentes que hace bien poco no eran pobres y que hoy por los efectos de la crisis, el paro y diferentes circunstancias han caído en la pobreza real"63. Un ejemplo significativo de lo que acabo de indicar lo encontramos en Francia, donde en el perío do comprendido entre 1984 y 1994 se incrementó considerablemente el número de pobres entre los desempleados (se pasó de un 32 a un $39 \%$ ), así como también hubo un incremento de la pobreza entre los grupos de edad más jóvenes y los asalariados con trabajos precarios, mientras que por el contrario se produjo un descenso entre los pensionistas (pasando de un 14 a un $7 \%{ }^{64}$.

\section{Recapitulación final}

El objetivo de una política progresista en el ámbito europeo debe ser el de lograr que haya más empleo para todas y todos, sin diferencias por razón de sexo y con aceptación del principio deigualdad en todas las políticas sociales y económicas, y que aquel sea estable y de calidad y que tome en considera-

son suficientes a menudo para hacer salir a los hogares de una situación de renta escasa".

Commission Europénne. Construire..., ob. cit.

63 F. J. Alonso, "Reflexiones sobre la pobreza y la exclusión social en España: nuevas formas y nuevas respuestas». Sistema, no 137/1997, pág. 56. Subraya también el autor la necesidad de prestar especial atención a las poblaciones infantiles y juveniles empobrecidas de forma creciente "porque ese es el presente más grave y el futuro más preocupante" (pág. 60).

64 INSEE, Économie et statistique, núms. 308, 309 y 310/1997. Se argumenta en el estudio citado que el incremento de la pobreza entre los desempleados se debe a varias razones, tales como «el incremento del desempleo de larga duración, la inestabilidad en el empleo de los jóvenes y las reformas que han endurecido las condiciones de acceso al seguro de desempleo». 
ción los intereses y las necesidades de las diferentes generaciones de trabajadores; al mismo tiempo, que se potencie dicha creación en sectores y ámbitos más vinculados a las necesidades de to das las personas con lo que ello implica también de revisión delas propias nociones de trabajo y del valor económico de una actividad ${ }^{65}$, y que se apueste claramente por políticas de apoyo a la educación, innovación tecnológica y a la formación del capital humano para poder asumir todo tipo de responsabilidades, así como por la reducción incentivada y negociada del tiempo de trabajo en la vida de cada persona y ampliando el abanico de posibilidades de distribución de dicho tiempo; también que se reformen los sistemas fiscales en orden a posibilitar la creación de empleo ${ }^{66}$, en la línea de luchar contra todo tipo de exclusión.

Todo ello, teniendo presente que la Europa comunitaria no vive aislada, ni mucho menos, del resto del mundo, y que por consiguiente debe participar de forma clara y decidida en el proceso actual de globalización económica, aportando su voz para defender políticas de protección social adecuadas, una regulación del sistema financiero que evite las convulsiones económicas vividas en los últimos tiempos y unas instituciones internacionales que estén a la altura de las necesidades del tiempo actual y que puedan tomar decisiones comprometidas en los plazos más breves posibles. Más y mejores empleos, en especial para los colectivos como los jóvenes, mujeres y desempleados de larga duración, no es en modo alguno incompatible con políticas económicas que aseguren el crecimiento y la competitividad, pues el objetivo de las instituciones comunitarias debe ser apostar por políticas que beneficien a la mayoría de la población. En el ámbito jurídico deberá extraerse toda la potencialidad posible a los derechos sociales incorporados al Tratado de Amsterdam y reforzar la intervención de las políticas comunitarias en el desarrollo de las redes de infraestructuras europeas, y en los ámbitos administrativos, la participación de las autoridades locales y regio-

65 En un importante estudio realizado para el Club de Roma se defiende esta tesis, argumentándose que "la misma noción de empleo, es decir de trabajo remunerado, no es más que una parte, aunque sea importante, de lo que deben considerarse actividades productivas", y que sólo una parte cada vez menos importante de dicho trabajo, que en la actualidad sería del 20 \% en los países avanzados, "está to davía ligado a actividades estrictamente manufactureras". O. Gianini y P.M. LiedtKe, El dilema... ob. cit, págs. 23 y 35.

66 En esta línea se manifiesta el Comité Económico y Social europeo, que apuesta por "una reducción de los costes salariales adicionales mediante un aumento de los impuestos indirectos, especialmente en el impuesto sobre el valor añadido y los impuestos sobre la energía". Dictamen. “Empleo y euro". DOCE C407, 28.12.98, pág. 285. 
nales en el diseño y puesta en práctica de las nuevas políticas sociales deviene del todo punto necesario y conveniente. En suma, la estrategia europea por el crecimiento y el empleo debería basarse en tres ejes: conseguir un crecimiento sostenible, mejorar el potencial de innovación y crecimiento, y lograr una sociedad más activa y con un mayor grado de inclusión social.

La concreción práctica de estas tesis debe manifestarse en el desarrollo del pacto europeo por el empleo aprobado en la cumbre de Colonia de los días 3 y 4 de junio de 1999, que sitúe a éste en el mismo plano de importancia que la estabilidad y el crecimiento económico, y que permita elevar la tasa de empleo hasta el $65 \%$ y en la perspectiva de llegar a medio plazo (año 2010) hasta el $70 \%$, reducir el desempleo al $4 \%$ y aumentar hasta el $60 \%$ la población activa femenina, así como reducir el porcentaje de personas que viven en situación de pobreza relativa hasta el $10 \%$. Ello requiere coordinar adecuadamente las políticas presupuestarias de los distintos Estados y conseguir que la política monetaria en manos del Banco Central Europeo se oriente a favor deesta estrategia,-sin olvidar las dificultad es existentes tanto en el terreno práctico como en el teórico, pues no conviene olvidar que el Tratado de Amsterdam le asigna como tarea principal "mantener la estabilidad de los precios"- así como establecer vías institucionales que permitan identificar a tiempo los cambios que se operan en el mundo industrial y así poder prever las medidas de respuesta adecuadas para amortiguar el impacto social. Junto a este pacto institucional los agentes sociales deberían negociar políticas salariales que tuvieran en consideración el marco europeo y las especificidades de cada Estado, negociaciones que no deberían significar en modo alguno, tal como defiende la Confederación Europea de Sindicatos y cuya tesis compartimos plenamente, "una carrera a la baja" salarial en los distintos Estados, y que además deberían incluir cuestiones tan relevantes como "Ios aspectos del reparto de las rentas, de la organización del trabajo y del tiempo de trabajo, de los cambios estructurales y de la seguridad social" 67 .

67 Memorandum a la Presidencia alemana del Consejo de Ministros. Bruselas, 28 de enero de 1999. 